\title{
Circulation and Topological Control in Image Segmentation
}

\author{
Luis Gustavo Nonato, Antonio M. da Silva Junior, \\ João Batista, and Odemir Martinez Bruno \\ Universidade de São Paulo, ICMC, São Carlos, SP, C.P. 668, 13560-970 Brazil \\ \{gnonato, antonio, jbatista, bruno\}@icmc.usp.br \\ http://www.icmc.usp.br
}

\begin{abstract}
In this paper we present an image segmentation technique based on the concepts of circulation and topological control. Circulation is a mathematical tool widely used for engineering problems, but still little explored in the field of image processing. On the other hand, by controlling the topology it is possible to dictate the number of regions in the segmentation process. If we take very small regions as noise, the mechanism can be seen as an efficient means for noise reduction. This has motivated us to combine both mathematical tool in our algorithm. As a result, we obtained an automatic segmentation algorithm that generates segmented regions bounded by simple closed curves; a desireable characteristic in many applications.
\end{abstract}

\section{Introduction}

Segmentation plays an important role in image processing especially for edge and object detection, coding and analysis. The spectrum of applications in which segmentation is to be found is quite wide, ranging from medical imaging to robot vision. Over the years a great number of approaches have been proposed, led by the fact that the efficiency of segmentation methods are heavily domain-oriented: the particularities of a problem found in a certain domain may demand the development of techniques with characteristics that are not necessarily suitable for other domains.

In this work we present a new segmentation technique that automatically decomposes an image into a set of regions whose boundaries are Jordan's curves, while keeping the topology of these regions under control. Three-dimensional reconstruction and object recognition (in which the topology of the object under investigation is normally known and where simple closed curves - Jordans curves - bounding the regions can be handled geometriacally) are examples of applications where such feature is desirable.

Making use of a vector field derived from image data, our approach employs the concept of circulation for such a field to decide which adjacent regions must be glued, as expected in region growing methods. The gluing is conducted by a mathematical framework capable of controlling the topology during the entire process. 
A consequence of the methodology above is that the segmentation process can be controlled by thresholding the circulation between adjacent regions as well as by the topological properties of the objects in the image. This flexibility makes our approach an interesting segmentation technique, which can be useful in many applications.

This paper is organized as follows: section 2 presents a brief description of prior work in image segmentation. In section 3 we review definitions and some properties necessary to figure out the next sections. The theoretical background for our algorithm is described in section 4. The algorithm itself is presented in section 5 . Results are discussed in section 6. Section 7 contains our conclusions and further work.

\section{Related Work}

The problem of image segmentation has received considerable attention in the literature [1216. Several methodologies have been proposed to tackle this problem, and the majority of them fall into two major approaches widely used in this context: edge-based-like 87/15] and region-based-like 3].

Region-based segmentation methods group pixels of similar properties (specific to a particular application domain), providing closed regions, which in turn give the boundaries. In edge-based approaches, on the other hand, discontinuities are extracted and the segmentation is guided by contours. The two approaches are complementary, and one may be preferred to the other for some specific applications or domain.

Compared to region-based segmentation techniques, however, edge detection has some very appealing properties. Usually, the algorithms are based on derivative calculations and can be implemented as a simple control structure and regular operators like convolution and, thus, lend themselves to an efficient implementation on special purpose image processors and parallel computers. In addition, edge detection techniques are able to localize surface boundaries more precisely in general.

In this paper, we add three different "functional views" or "perspectives" in which image processing techniques could be categorized, according to the role played in the application domain. They are: degree of automation, topological control and local/global information usage.

Under the first perspective, image processing techniques are classified according to the degree of automation provided. For some domains, like medical imaging, user-free segmentation techniques are highly desirable, as some modalities (CT, MRI) produce multidimensional data sets and require the interpretation of various slices. From this perspective, several semi-automatic and automatic methods for segmenting images have been proposed. Semi-automatic methods are those which require some degree of information, usually entered by the user interactively, either in the beginning or during the process. Various classes of algorithms fall into this category. One typical example are the deformable models such as snakes [5], in which an initial snake (mostly outlined manually around 
an object of interest) is deformed under internal (bending) and external forces (lines and edges, mainly), converging to a final form to reach an equilibrium energy state.

On the other hand, automatic methods do not require user interaction. Some edge- and region-based approaches can be categorized according to this functionality. Signal Processing techniques (Fourier Transform [1], Wavelets [9]) and some statistical methods - if one considers segmentation as a pixel classification problem - such as Fuzzy [13] and Clustering [4, can lead to segmentation with no human intervention.

A second, but nonetheless important perspective, is the control over the image topology while carrying out segmentation. Few methods are to be found in the literature that are capable of, simultaneously, keeping track of topology while splitting an image into regions of interest. In practical terms, by controlling the topology one can dictate the number of nested segmented regions. This is a very desirable feature for certain domains like segmentation of medical images in which different anatomical structures are sought. For example, the segmentation of an axial image of the brain with Euler number equal to 1 could produce a single contour of the skull (assuming this is the most external anatomical structure present). But, if Euler number is set to values smaller than 0, other internal structures (along with the skull itself) would appear as the result of the segmentation 11 .

The third perspective takes into account the usage of either local or global information to reach segmentation. Local information based algorithms use the pixel neighborhood and pixel connectivity as input of the segmentation process. Several families of algorithms belong to this class: classic edge detection [8], mathematical morphology [14], convolution-based techniques, etc. Algorithms based on global information, on the other hand, consider information from an image region or a larger set of pixels, as opposed to a pixel and its near neighborhood. In general, approaches based on global information are used on region segmentation, texture algorithms and active contours.

Both local and global information-based approaches hold important information of the image nature and are functionally complementary. Techniques that explore both local and global features may be very promissing. Methods based on Markov Random Fields (MRF) [6 2, for example, fall in this category. MRF models represent an image through local characteristics, by defining the dependency of each pixel value with its neighbouring pixels. This dependency is expressed in terms of a conditional probability defined globally.

To our knowledge there is no method that combines the three functional view altogether. The majority of the techniques available concentrate on a single view alone and some combines two of them. The method described in this paper encompasses characteristics from the three functional views presented above. It is an automatic approach with full control over topology and, moreover, combines both local and global information. 


\section{Basic Concepts}

This section presents the basic definitions and notation that will be used throughout this paper. The approach undertaken here has been restricted to the twodimensional Euclidean space $\mathbb{R}^{2}$.

A cell with center $(a, b)$ and radius $q$ is the set of points $(x, y) \in \mathbb{R}^{2}$ satisfying $\max (|x-a|,|y-b|) \leq q$, i.e., a square with side length $2 q$ centered in $(a, b)$. The corners of a cell are called vertices of the cell and the four segments bounding the cell are its edges.

A square grid $\mathcal{G}$ is a cell decomposition of $\mathbb{R}^{2}$ where each point $(a, b)$, where $a$ and $b$ are integers, is the center of one single cell $V$ (grid cell) with radius equal to $\frac{1}{2}$.

A finite subset of grid cells $R$ is a region of $\mathcal{G}$ if for any two cells $V_{a}$ and $V_{b}$ in $R$ there is a sequence of grid cells $\left(V_{1}, \ldots, V_{n}\right)$ in $R$ such that $V_{a}=V_{1}$, $V_{b}=V_{n}$, and $V_{i} \cap V_{i+1}$ contains a common edge of $V_{i}$ and $V_{i+1}$. From this we can see that each edge in $R$ is contained in either one or two cells of $R$, which are called boundary edges and interior edges, respectively. Note from the definion above that each region is a 4-connected set of cells.

Two regions $R$ and $S$ are called adjacent regions if $R \cap S=\sigma$, where $\sigma$ is a set of boundary edges.

Let $\gamma=\left(e_{1}, \ldots, e_{n}\right)$ be a sequence of distinct boundary edges of a region $R$ such that $e_{i}$ and $e_{i+1}\left(e_{n+1}=e_{1}\right), i=1, \ldots, n$, have a vertex in common. $\gamma$ is said to be a external boundary curve of $R$ if $\gamma$ encloses $R$ and as one "walked" from edge $e_{i}$ to $e_{i+1}$, the cells (or cell) in $R$ containing $e_{i}$ and $e_{i+1}$ are always located on the left of these edges. If $\gamma$ is enclosed by $R$ and the cells containing its edges are always to the right of the edges, then $\gamma$ is called a hole of $R$. With the definitions above we stipulate a counter-clock-wise orientation for the boundary of the regions in $\mathcal{G}$, which is essential for the Green's theorem described in next section. The union of the external boundary curve with the holes of $R$ is called the boundary curve of $R$, denoted $B_{d}(R)$.

Let $\mathcal{U}$ be a subset of $\mathcal{G}$ such that the center $(a, b)$ of each cell in $\mathcal{U}$ satisfies $1 \leq a \leq N$ and $1 \leq b \leq M$. An $N \times M$ digital image, denoted $\mathbb{I}$, is a pair $(\mathcal{U}, I)$, where $I: \mathcal{U} \rightarrow \mathbb{R}^{+}$is a function that associates each grid cell in $\mathcal{U}$ with a positive value. Note that in our context a digital image can be seen as a set of cells with scalars associated with them.

Let $n c(R)$ and $n h(R)$ be the number of connected components and holes of a region $R \subset \mathcal{G}$, respectively. The Euler characteristic of $R$ can be defined by

$$
\chi(R)=n c(R)-n h(R) .
$$

It is worth mentioning that the Euler characterisctic is usually defined either in terms of the number of vertices, edges, and faces or as a difference between the number of connected components and the number of holes in an object. As will be shown in the next section, this last definition is more appropriate in the context of this work. 


\section{Circulation and Topological Control}

In this section we describe the mathematical framework that is the background of our segmentation algorithm. Such a framework is based on two main concepts, namely: circulation through boundary curves and topological control during the gluing process. These concepts are detailed in the following subsections.

\subsection{Circulation Through Boundary Curves}

Let $R$ be a region in a digital image $\mathbb{I}=(\mathcal{U}, I)$, i.e., each cell in $R$ is associated with a positive scalar, and $E$ the set of the edges in $R$. Let $F_{R}: E \rightarrow \mathbb{R}^{2}$ a map that relates each edge $e \in E$ to a two-dimensional vector $F_{R}(e)=(p, q)$, where $p: \mathcal{N}_{R_{e}} \rightarrow \mathbb{R}$ and $q: \mathcal{N}_{R_{e}} \rightarrow \mathbb{R}$ are real functions from a neighborhood $\mathcal{N}_{R_{e}}$ of $e$, in $R$, to $\mathbb{R}$. Notice that $F_{R}$ is a vector field defined in $R$. It is worth mentioning that the vector $F_{R}(e)$ will depend on the arrangement of the cells in the neighborhood of $e$ as well as the escalar values of these cells.

Proposition 1 below states an important result that is essential for our regionbased segmentation algorithm.

Proposition 1. If $F_{R}: E \rightarrow \mathbb{R}^{2}$ is constant, i.e., $p=c_{1}$ and $q=c_{2}$, for all edge in $R$ then

$$
\oint_{B_{d}(R)} F_{R} \mathrm{~d} s=0
$$

Proof. The proof follows from Green's theorem, as

$$
\oint_{B_{d}(R)} F \mathrm{~d} s=\iint_{R} \frac{\partial q}{\partial x}-\frac{\partial p}{\partial y} \mathrm{~d} x \mathrm{~d} y
$$

and $\frac{\partial q}{\partial x}=0, \frac{\partial p}{\partial y}=0$ for all edges in $R$.

Proposition 1 deserves some comments. Although Green's theorem is usually defined in the context of continuous vector fields, there are different versions of such a theorem for the discrete case (see [18]). With some manipulation, the proof of Proposition 1 can similarlly be carried out with a discrete version of Green's theorem.

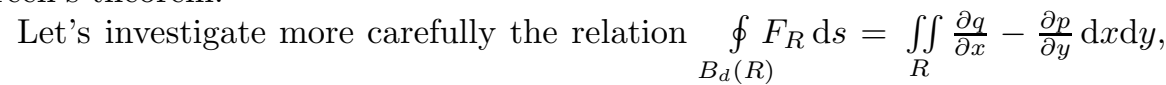
given by Green's theorem in the proof of Proposition 1, The term $\frac{\partial q}{\partial x}-\frac{\partial p}{\partial y}$ on the right double integral represents the $z$ component of the rotational vector of $F$ and it measures the circulation of $F_{R}$ in each point of the domain. The important fact is that circulation can be seen as a high-pass filter when $F_{R}$ is properly defined, as illustrated in figure 1. Figure 1 b) shows the circulation of figure 1a) for $F_{R}=(p, q)$ defined as $p=0.3 I_{i j}+0.25\left(I_{i+1 j}+I_{i-1 j}\right)+0.1\left(I_{i j-1}+I_{i j+1}\right)$, $q=0.3 I_{i j}+0.25\left(I_{i j-1}+I_{i j+1}\right)+0.1\left(I_{i+1 j}+I_{i-1 j}\right)$ (we are supposing that $R$ 
a)
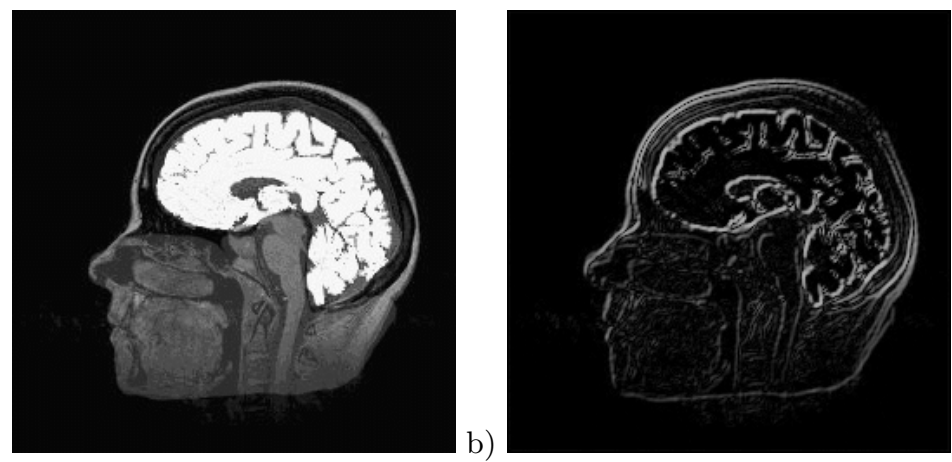

Fig. 1. Circulation as a high-pass filter

is the whole image). Notice from figure 1b) that the high-frequence areas of the image in figure 1a) could be well detected by measuring the circulation of $F_{R}$.

Hence, in low-frequence areas, the integral $\iint_{R} \frac{\partial q}{\partial x}-\frac{\partial p}{\partial y} \mathrm{~d} x \mathrm{~d} y$ will assume values close to zero, the same happening with $\underset{B_{d}(R)}{\oint} F_{R} \mathrm{~d} s$. Thus, by analyzing this last integral we can have an indication whether the region $R$ is crossing or not a high-frequence area. This is an essencial matter in image segmentation. Regions where the integral $\oint F_{R} \mathrm{~d} s$ is equal to zero are named homogeneous regions. $B_{d}(R)$

Notice that regions where $F_{R}$ is constant are always homogeneous. This fact will be important in the development of the segmentation algorithm presented in the next section.

Let $R$ and $S$ be two adjacent regions in a digital image $\mathbb{I}=(\mathcal{U}, I)$ and $\sigma=R \cap S$ be the intersection curve between $R$ and $S$. In order to analyze the circulation in $\sigma$ we need to define the vector field in the edges of $\sigma$. A natural way to do this is define $F_{\sigma}=(p, q)$ so that the components $p$ and $q$, for each edge $e \in \sigma$, are real functions from a neighborhood of $e$ in $R \cup S$.

Next proposition, which is a consequence of the discussion above, tell us how to glue homogeneous regions while keeping the homogeneity.

Proposition 2. Let $R$ and $S$ be adjacent homogeneous regions and $F_{\sigma}=(p, q)$ the vector field defined in $\sigma=R \cap S$ as discussed above. If $\frac{\partial q}{\partial x}-\frac{\partial p}{\partial y}=0$, for each edge in $\sigma$, then $R \cup S$ is also homogeneous.

\subsection{Topological Control}

In this subsection we shall investigate how to characterize the topology of the union of two adjacent regions. More specifically, we are interested in identifying the Euler characteristic of $R \cup S$ where $R$ and $S$ are two adjacent regions whose topologies are given by $\chi(R)$ and $\chi(S)$, respectively.

In section 2 we defined the Euler characteristic of a region $R$ in terms of its number of connected components and holes, i.e., $\chi(R)=n c(R)-n h(R)$. As in 
our context regions are always constituted by single connected component, if we characterize the number of holes in $R \cup S$, we shall identify its topology.

Before presenting such a characterization, let us understand the topological behavior of curves generated by intersecting two adjacent regions. If $R$ and $S$ are two adjacent regions then either $S$ is inside $R$ (or vice-versa) or $R$ and $S$ are side by side, as shown in figure 2 . In the former, the intersection curve consists in a simple closed curve, as illustrated in figure 2a). Curves generated by intersecting side by side adjacent regions can be formed by a set of disjoint segments. For example, in figure 2 $\mathrm{b}$ ), the intersection between the adjacent regions gives rise to a curve with two connected components.
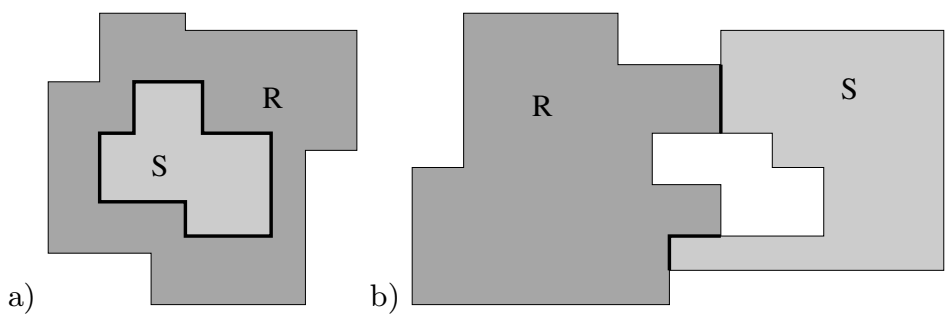

Fig. 2. Intersection of adjacent regions generating: a) a simple closed curve, b) a set of curve segments

Hence, supposing that $\sigma=R \cap S$ is the intersection curve between $R$ and $S$, we can also compute the Euler characteristic of $\sigma$ as $\chi(\sigma)=n c(R \cap S)-n h(R \cap S)$, where $n c(R \cap S)$ and $n h(R \cap S)$ are the number of connected components (or segments) and holes in $R \cap S$, respectively. Notice that $n h(R \cap S)$ can only assume value 1 if $S$ is inside $R$ (or $R$ is inside $S$ ). Otherwise, $n h(R \cap S)$ becomes 0 . Furthermore, if $n h(R \cap S)=1$ then $n c(R \cap S)=1$.

Next proposition allows us to quantify, in terms of $\chi(\sigma)$, the number of new holes created when two adjacent regions are unified. We denote this number of new holes by $n h_{\text {new }}(R \cup S)$, i.e., $n h_{\text {new }}(R \cup S)=n h(R \cup S)-n h(R)-n h(S)$.

Proposition 3. Let $R$ and $S$ be two adjacent regions and $\sigma=R \cap S$ their intersection curve. The number $n h_{\text {new }}(R \cup S)$ of new holes generated by gluing $R$ and $S$ is:

$$
n h_{\text {new }}(R \cup S)=\chi(\sigma)-1
$$

Proof. We know that

$$
n h_{\text {new }}(R \cup S)=n h(R \cup S)-n h(R)-n h(S)
$$

Additionally, we have that $\chi(R \cup S)=\chi(R)+\chi(S)-\chi(\sigma)$, which from equation [1] becomes

$$
n h(R \cup S)=n c(R \cup S)-n c(R)+n h(R)-n c(S)+n h(S)+\chi(\sigma)
$$


Substituting equation above in (2) and remembering that $n c(R \cup S)=n c(R)=$ $n c(S)=1$ we conclude the proposition.

In the next section we show how the mathematical framework presented above can be handled in order to suit image segmentation effectively.

\section{Algorithm}

The segmentation algorithm proposed in this work can be divided in three parts, namely: vector field definition, initialization, and region growing. The following subsections are devoted to detailing each of these parts.

\subsection{Vector Field Definition}

The vector field plays an essential part in our algorithm, as it dictates the behavior and the quality of the segmentation process. Notice that different vector fields can produce distinct results. In our implementation the vector field is defined from weighted mean values in the neighborhood of each edge. More specifically, let $R$ be a region in a $N \times M$ digital image $\mathbb{I}=(\mathcal{U}, I)$ and $E$ the edges of $R$. We define the vector field $F_{R}=(p, q)$ as follows:

$$
\begin{aligned}
& p(e)=\frac{1}{C} \sum_{V_{i} \in \mathcal{N}_{R_{e}}} c_{i} I\left(V_{i}\right) \\
& q(e)=\frac{1}{D} \sum_{V_{i} \in \mathcal{N}_{R_{e}}} d_{i} I\left(V_{i}\right)
\end{aligned}
$$

where $c_{i}$ and $d_{i}$ are constants satisfying $c_{i}, d_{i}>0, \forall i$ and $C=\sum_{\mathcal{N}_{R_{e}}} c_{i}, D=$ $\sum_{\mathcal{N}_{R_{e}}} d_{i}$. It is important to note that $C$ and $D$ depend on the number of cell in $\mathcal{N}_{R_{e}}$. The values of $c_{i}$ and $d_{i}$ are composed by applying a mask to each edge $e$. Figure 3 ) and $3 \mathrm{~b}$ ) shows the masks for horizontal and vertical edges, which define the values for $c_{i}$ and $d_{i}$, respectively.

If $e$ is either a boundary edge or an edge close to the boundary of $R$, the values of $c_{i}$ and $d_{i}$ are specified by intersecting the mask with $R$. Figure 4 shows two examples of such an intersection. Notice that the normalization factors $C$ and $D$ are computed as a sum of the mask values in the intersection.

\subsection{Initialization}

The initialization step aims at starting the segmentation process with a set of regions satisfying proposition [1, i.e., $F_{R}$ must be constant in each edge of the inicial regions, implying that the line integral of $F_{R}$ on the boundary curve of each region $R$ is equal to zero. 
a)

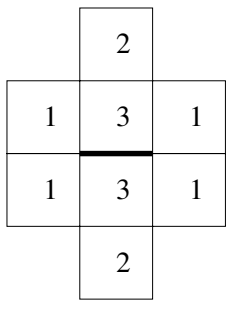

b)

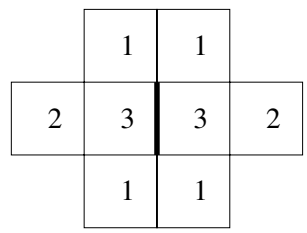

Fig. 3. a) Values of $c_{i}$; b) values of $d_{i}$

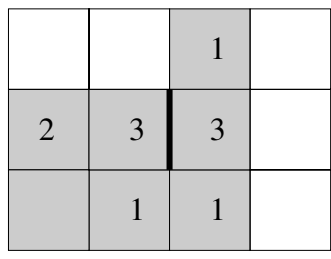

$\mathrm{C}=11$

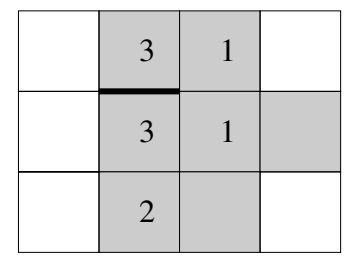

$\mathrm{D}=10$

Fig. 4. Examples of the intersection between the mask (applyed in the bold edges) and a region $R$ (gray cells)

An easy way to create these initial regions while ensuring proposition 1, is to make use of the grid cell comprising the whole image as the initial regions. Moreover, since each grid cell of the image is associated with a single scalar, the vector field $F_{R}$ is constant, thus ensuring proposition 11. The main reason for restraining $F_{R}$ to be a constant in each initial region is that this property guarantees the homogeneity, i.e., such regions are not crossing a high-frequence area.

After initializing the regions, we compute and store, in a priority queue, the values (and edges) of $\frac{\partial q}{\partial x}-\frac{\partial p}{\partial y}$ evaluated on the boundary edges. The priority queue stores the elements in increasing order and it is used in the growing process to decide which regions must be merged, as discussed in next subsection.

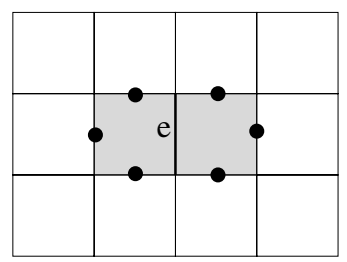

Fig. 5. Least square approximation takes into account the values of $p$ and $q$ in the marked boundary edges 
Before presenting the growing process, it is important to discuss how to estimate the derivatives $\frac{\partial q}{\partial x}$ and $\frac{\partial p}{\partial y}$. In our implementation we are making use of least square interpolation to compute second order polynomials from which the derivatives are computed. The least square approximation generates a second order polynomial for each component $p$ and $q$ of the vector field in each boundary edge $e$, taking into account the values of $p$ and $q$ from the edges of the cells adjacent to $e$. Figure 5 illustrates which are the the values of $p$ and $q$ involved in the calculation of the polynomials in an edge $e$.

\subsection{Region Growing}

The region growing process makes use of the values stored in the priority queue to decide which regions must be glued. The regions adjacent to an edge extracted from the priority queue are merged and the boundary curve of the new region is updated. In order to continue with the growing process it is necessary to compute the circulation on the new boundary curve.

Inspired by proposition 2, we estimate the circulation in each new component of the boundary curve by computing the scalar field line integral

$$
\oint_{B_{d}(R \cap S)}\left|\frac{\partial q}{\partial x}-\frac{\partial p}{\partial y}\right| \mathrm{d} l
$$

where $R$ and $S$ are regions that become adjacent after gluing. The computed value is also inserted into the priority queue. For example, suppose that $R_{1}$ and $R_{2}$ are the regions selected to be merged, as shown in figure [6]). After gluing $R_{1}$ and $R_{2}$, the new boundary curve component (highlighted as bold in figure 6b)) is updated and the scalar field line integral (5) is computed on it, and the computed value is stored into the priority queue. Therefore, the region growing process aims at merging the regions in an order that preserves homogeneity.

a)

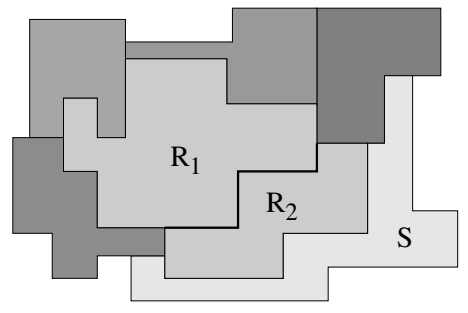

b)

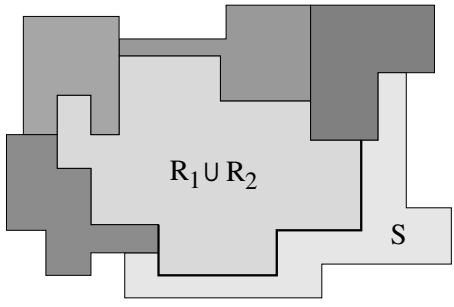

Fig. 6. Keeping the homogeneity in the growing process

The region growing process will have control over the region's topology if proposition 3 is employed, as it allows us to know if new holes are been created during the gluing process. Hence, it is possible to specify, for example, a maximal number of holes in each region. It is also possible to control the size of the holes 
in a straightforward way. In fact, it is well known that Green's theorem allows us to compute the area of a region through the line integral of the vector field given by $F=(-y, x)$, or similiarly, by computing the summation

$$
\frac{1}{2} \sum_{i}^{n} v_{x}^{i} v_{y}^{i+1}-v_{y}^{i} v_{x}^{i+1}
$$

where $v_{x}^{k}$ and $v_{y}^{k}$ are the components $x$ and $y$ of the vertices $v^{k}$ of a poligonal curve (boundary curve).

As a result, we can estimate the areas of each new hole created by the gluing operation, discarding the ones whose areas are below a desired value. Notice that this procedure can be employed as an alternative tool to noise reduction.

In our implementation we employ two different stopping criteria for the growing region process. The first one ends the process by thresholding the values of the line integral (5). That is, when the priority queue returns a value higher than a threshold, the region growing process stops.

The second stopping criterion takes into account the number of detected regions. In this case the region growing process continues until a desired number of regions is obtained. In our implementation, this criterion does not consider the background of the image as a valid region.

\section{Results}

In this section we present some results obtained from the framework shown above. The axial MRI image of the brain shown in figure 7 has been used to illustrate our algorithm.

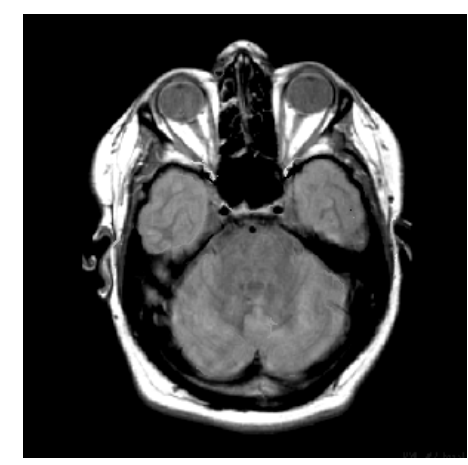

Fig. 7. Axial MRI image used in the segmentation

As mentioned in the previous section, our algorithm considers two different stopping criteria: the line integral thresholding (Eq. (5) and topological properties thresholding. Figure 8 shows the boundary curve of the regions detected by 
thresholding line integral (5). In this case, the resulting segmentation contains 400 regions approximately and some of them can be accounted on noise. Such noisy regions are not easily removed, since finding an appropriate threshold value to remove them may be a difficult task.

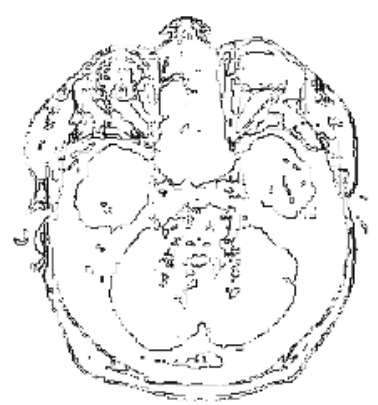

Fig. 8. Boundary curves of the regions dected by thresholding the line integral (5)

The difficulty in removing noisy regions can be overcome by topological thresholding. Some examples are shown in figure 10, Figure [10a) shows the resulting boundary curve when the topological threshold is set to a single region without holes (we are not taken into account the background region). Note that the curve that bounds the head is well detected and the small regions, which are caracterized as holes, are eliminated altogether. Figure 10 b) presents the resulting regions (boundary curves) when the topological threshold is set to a single component with a single hole. Figure 10k) shows the resulting regions obtained by setting the number of components to one, the number of holes inside this component to one, and the number of holes within the hole equal two. As the holes are indexed during the growing process, it is straightforward to select some of them in accordance with a desired criterion. In our implementation the holes are chosen from the area, i.e., the holes with the largest areas are selected.

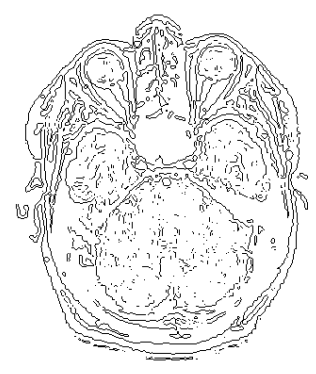

Fig. 9. Segmented image by zero-crossing of the LoG with $\sigma=1.5$ 
Figure 9 depicts an edge image obtained by the classical zero-crossing of the Laplacian of the Gaussian (LoG) (with $\sigma=1.5$ ). Although this segmentation looks similar to that generated by the proposed method, it does not deal with topological control and, therefore, can not guarantee that edges are closed curves, which define a single region. The topological control provided by the proposed method turns out to be an efficient mechanism to keep noise under control, since the number of regions in the resulting image is defined by a set of parameters when the segmentation begins. This behavior can be observed in figures 8 and 10 .

a)

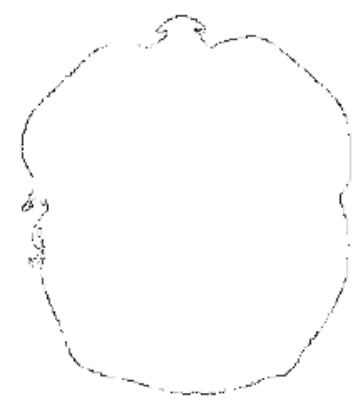

c)

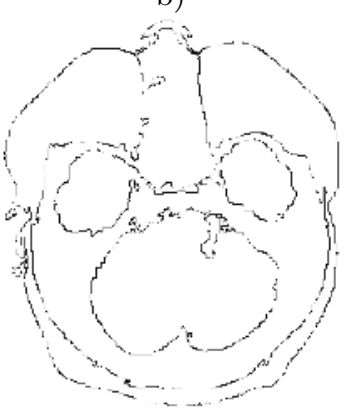

Fig. 10. Regions (and their boundary curves) dected by thresholding the topological properties as: a) a single component; b) a single component with a hole; c) a single component with a hole which has other two holes

To process the image in figure 7 , the algorithm took $1.07 \mathrm{~s}$ for the initialization step and $1.77 \mathrm{~s}$ for the region growing (with topological control) stage on a $\mathrm{P} 4$ $2.4 \mathrm{GHz}$ and $512 \mathrm{MB}$ RAM. This is a satisfactory result when compared with other automatic segmentation techniques described in the literature.

An important property of the algorithm, which can be observed in figure 10 . is that the boundary curves produced are Jordan's curves, which are adequate for $3 \mathrm{D}$ reconstruction from contours as discussed in [10. Such feature is not commonly found in other segmentation algorithms. 


\section{Conclusions and Future Work}

This paper presented a new framework to image segmentation that makes use of circulation and topological control during the region growing process. From such a framework we derived an automatic segmentation algorithm capable of detecting regions while keeping their boundaries as Jordan's curves; a desirable property in many applications. The built-in topological control of the algorithm has proven to be an efficient mechanism to reduce noise and enhance the quality of the segmented regions.

The framework is also flexible, as different vector fields (from which the circulation is computed) may produce different segmentations. In fact, this subject is currently under investigation as we are now working on defining a vector field to segment images with texture. We are also investigating how the topological control can be used as a matching creterion. By imposing a certain number of holes in a segmentation process for a single image, we can detect a set of other images with similar characteristis, that is, those which holes are similar in shape or area, for example.

\section{Acknowledgments}

We aknowledge Brazilian Funding Agencies FAPESP (proc. \# 03/02815-0) and CNPq (proc. \# 307268/2003-9) for supporting this work.

\section{References}

1. E. Brigham. Fast Fourier Transform and its applications. Englewood Cliffs, NJ: Prentice-Hall, 1988.

2. R. Chellappa and A, Jain (eds.). Markov Random Fields. Academic Press, 1993.

3. X. Jiang. An Adaptive Contour Closure Algorithm and Its Experimental Evaluation. IEEE Trans. Patt. Anal. Mach. Intell., vol 22, no 11, 2000.

4. Kanumgo. An Efficient k-Means Clustering Algorithm: Analysis and Implementation. IEEE Trans. Pattern Anal. Machine Intell., vol. 24, no. 7, pp. 881-892,2002.

5. M. Kass. A. Witkin and D. Terzopoulos. Snakes: Active Contour Models. Int. Journal of Computer Vision, 312-331, 1988.

6. S. Z. Li. Markov random field modeling in computer vision. Springer-Verlag, 1995.

7. Y. Lin, J. Dou, and E. Zhang. Edge Expression Based on Tree Structure. Pattern Recognition, vol. 25, no. 5, pp. 507-517, 1992.

8. W. Ma and B. Manjunath. EdgeFlow: A Technique for Boundary Detection and Image Segmentation. IEEE Trans. on Image Processing, vol. 9, no. 8, 2000.

9. S. Mallat. A Wavelet Tour of Signal Processing, 2nd Edition. Academic Press, 1999.

10. L.G. Nonato, R. Minghim, M.C.F. Oliveira and G. Tavares. A novel approach to Delaunay 3D Reconstruction with a comparative analysis in the light of applications. Computer Graphics Forum., v. 20, n. 2. p. 161-174, 2001.

11. L.G. Nonato, R. Minghim, A. Castelo, J. Batista. Morse Operators for Digital Planar Surfaces and their Application to Image Segmentation. IEEE Transactions on Image Processing, v. 13, n. 2, p. 216-227, 2004. 
12. N. Pal and S. Pal. A review on image segmentation techniques. Pattern Recognition, 26: 1277-1294, 1993.

13. M. Rezaee et al. A Multiresolution Image Segmentation Technique Based on Pyramidal Segmentation and Fuzzy Clustering. IEEE Trans. on Image Processing, vol. 9, no. 7, 2000.

14. J. Serra. Image Analysis and Mathematical Morphology. Academic Press, New York, 1982.

15. B. Song and S. Lee. On the Range Image Segmentation Technique Based on Local Correlation. Proc. Second Asian Conf. Computer Vision, pp. 528-532, 1995.

16. M. Sonka et al. Image Processing, Analysis and Machine Vision. PWS Publishing, 1999.

17. C. Xu, D. L. Pham and J. L. Prince. Image Segmentation using Deformable Models. Handbook of Medical Imaging, vol. 2, chapter 3. SPIE Press, 2000.

18. L. Yang and F. Albregtsen. Fast and exact computation of Cartesian geometric moments using discrete Green's theorem. Pattern Recognition, Vol. 29, No. 7, pp. 1061-1073, 1996. 